\title{
PHENOTYPIC AND FUNCTIONAL CHARACTERIZATION OF CD4+ T-CELLS INFILTRATING THE CENTRAL NERVOUS SYSTEM OF RATS INFECTED WITH CORONAVIRUS MHV IV
}

\author{
H. Imrich, S. Schwender, A. Hein and R. Dörries \\ Institut für Virologie und Immunbiologie der Universität Würzburg \\ Versbacher Str. 7 \\ 8700 Würzburg,
}

\section{INTRODUCTION}

Mouse hepatitis virus strain JHM, a neurotropic member of coronaviridae, causes neurological diseases in rats after intracerebral inoculation. The course of the infection depends on the rat strain, the age of the animals and the type of virus used. After infection at the age of 3 weeks BN rats remain clinically healthy. In contrast, $40 \%$ of LEW rats die within the first week past infection, when inoculated at the same age. Most of the surviving animals develop neurological signs with increasing severity up to the second week past infection, followed by complete convalescence $1,2,3$.

Histopathologically, the infection is characterized by demyelinated areas along with infiltrating mononuclear cells. In clinically healthy $\mathrm{BN}$ rats demyelinated foci are small and localized around the ventricles, whereas in LEW rats wide-spread demyelination is seen, extending from brain to spinal cord ${ }^{1}$. Within the demyelinated areas differences can be detected between the two rat strains with respect to the amount of infiltrating lymphocytes as well as to the spatial arrangement of lymphocyte subpopulations. In LEW rats higher numbers of $\mathrm{CD} 8^{+} \mathrm{T}$-cells dominate the inflammatory response in the brain parenchyma early past infection 3 . In contrast, in $\mathrm{BN}$ rats $\mathrm{CD} 4^{+} \mathrm{T}$-cells constitute the major infiltrating cell type at any time past infection. Both, $\mathrm{CD} 8^{+}$and $\mathrm{CD} 4^{+} \mathrm{T}$-cells, are homing close to and in virus-infected areas suggesting that most of these cells are virus specific 3 . The humoral immune response is characterized by striking differences on a functional level between the two rat strains. BN rats recruit high numbers of virus-specific antibody secreting cells into the brain tissue in response to the viral infection. This is in contrast to Lewis rats where these cells appear later and in much lower numbers 5 . 
Because $\mathrm{CD} 4+\mathrm{T}$-cells play a key role in the differentiation process of B-cells, we present in this report our efforts to characterize CD4+ T-lymphocytes from the brain of infected LEW and BN rats with respect to their state of activation 6 days past infection (dpi), a time when the majority of JHM-infected LEW rats reveal the first signs of neurological disease $^{3}$.

\section{MATERIAL AND METHODS}

Virus. The JHM strain of coronavirus (MHV4) for infection of rats was propagated as described earlier ${ }^{5}$. Virus particles used as antigen were purified according to Wege et al. ${ }^{6}$.

Animals. LEW and BN rats were obtained at the age of 3 weeks from the "Zentralinstitut für Versuchstierzucht" (Hannover, FRG). They were inoculated with $1 \times 10^{3}$ plaque-forming units of JHM virus. Neurological signs of the infection were scored daily according to a scale published previously ${ }^{3}$.

Isolation of lymphocytes. Lymphocytes were isolated from cervical lymph nodes (CLN) by mincing the tissue through a stainless steel sieve into ice-cold Hank's buffer containing 3\% fetal calf serum (FCS). Lymphocytes were collected from brain and spinal cord by density gradient centrifugation after mechanical disruption and enzymatic digestion of CNS tissue ${ }^{5}$. In order to enrich T-cells the adherent cell fraction was removed from CNS isolated lymphocytes by passage through a nylon wool column.

Phenotyping of isolated lymphocytes. Surface markers of isolated lymphocytes were determined by double immunofluorescence and flow cytometric analysis as published previously 3 . The FITC-labeled monoclonal antibody (mab) W3/25 specific for the rat CD4 molecule was used either in combination with the mab OX22 (rat CD45RB) or the mab OX25 (rat interleukin 2 receptor) to stain lymphocytes. OX22 or OX25 mabs were detected on lymphocytes by phycoerythrin (PE)-labeled goat anti-mouse IgG antibodies. Primary antibodies were purchased from Serotec (Wiesbaden, FRG) and PE-labeled secondary antibody from Dianova (Hamburg, FRG).

Proliferation assay. Lymphocytes were cultured in round bottom 96 well microtiter plates for 72 hours (RPMI medium, $5 \times 10^{-5} \mathrm{M} 2$-mercapto-ethanol, 3\% normal rat serum). To monitor the proliferative activity $0,1 \mu \mathrm{Ci}$ of ${ }^{3} \mathrm{H}$-thymidine (Amersham Buchler, Braunschweig, FRG) were added per well at 48 hours of culture. Antigen- or mitogeninduced proliferation was assayed by addition of keyhole limpet hemocyanine (KLH) or 
JHM virus, both at a concentration of $20 \mu \mathrm{g} / \mathrm{ml}$ or concanavalin A (Con A) at a concentration of $4 \mu \mathrm{g} / \mathrm{ml}$ to the cells immediately after onset of cultures. Incorporation of tritiated thymidine was measured in a multi-chanel scintillation counter ( $\beta$-Plate, Pharmacia, Freiburg, Germany). Mean values were calculated from 2 or 3 wells.

\section{RESULTS AND DISCUSSION}

In a first approach to characterize the infiltrating CD4+ $\mathrm{T}$-lymphocyte population their phenotype was analyzed by flow cytometry. The majority of CD4+ T-cells isolated from the brain of both rat strains were CD45RB-, CD25- (data not shown). Loss of the CD45RB marker that is expressed on naive CD4+ $\mathrm{T}$-cells 7,8 indicated that the majority of $\mathrm{T}$-cells in the brain tissue was of the memory/primed phenotype. The low expression of CD25 (interleukin 2 receptor, IL2R) was surprising because presence of viral antigen in the brain of both rat strains up to 3 weeks past infection ${ }^{3}$ and upregulation of MHC antigens class II antigens on microglia cells ${ }^{9}, 10$ suggested that primed $\mathrm{CD}^{+}{ }^{+} \mathrm{T}$-cells could be stimulated in the CNS tissue to local proliferation leading to the typical CD4+ T-cell accumulation observed in the virus infected areas in the brain tissue ${ }^{3}$.

To investigate the question of T-lymphocyte expansion in the CNS more closely, leukocytes from the CNS were enriched for T-lymphocytes by nylon wool passage and assayed for DNA synthesis ex vivo by incorporation of ${ }^{3} \mathrm{H}$-thymidine. As can be seen in figure 1 the uptake of ${ }^{3} \mathrm{H}$-thymidine in these cells is not much higher compared to the background incorporation of T-cells from uninfected animals $(50$ to $80 \mathrm{cpm}$ ) indicating that the proliferative activity of inflammatory T-cells in the JHM-infected CNS was low in both rat strains. The weak proliferative response observed in $1 \times 105 \mathrm{CNS}$-isolated lymphocytes was confirmed by assaying 5 times more T-lymphocytes from the CNS (figure 1). Since we failed to stimulate proliferation of these cells with a strong T-cell mitogen like Con A (Fig. 2 , left) and JHM virus-specific antigens, even in the presence of irradiated feeder cells from secondary lymphoid organs, we tend to believe that primed T-cells entering the JHM virusinfected brain do not proliferate and are rendered insensitive to further stimulation of proliferation. As a consequence, increase of T-lymphocytes in the brain parenchyma of both rat strains as reported by us previously ${ }^{3}$ reflects rather transient retention and accumulation of these cells than antigen-driven expansion at the site of infection.

This hypothesis is strengthened by findings of Matsumoto et al., ${ }^{11}$ who reported absence of proliferating CD4 ${ }^{+} \mathrm{T}$-cells in the CNS of LEW rats suffering from experimental allergic encephalitis (EAE), an autoimmune reaction directed versus the myelin-containing glia cell fraction. Moreover, passive transfer of proliferating T-cells from EAE animals resulted in CNS inflammation but suppression of proliferation in the $\mathrm{CD} 4+$ lymphocyte population after entry into the CNS. Nelson and co-workers ${ }^{12}$ reported low cloning efficiency of 
T-lymphocytes isolated from lungs due to markedly reduced capacity of these cells to proliferate. Most likely lung-resident macrophages exert these suppressive effects on the nonrecirculating T-lymphocyte population in this organ. From these data it is conceivable that as a general principle expansion of CD4+ T-lymphocytes is not allowed in extra-lymphoid tissue. In this context, the immunologically sequestered situation of the unaffected CNS $^{9}$ rises the question for the extra-cerebral lymphoid tissue where priming and expansion of JHM-specific T-cells occur.

The deep cervical lymph nodes (CLNs) are known to be involved in the recruitment of a specific immune response to antigens that were administered to the ventricles of the

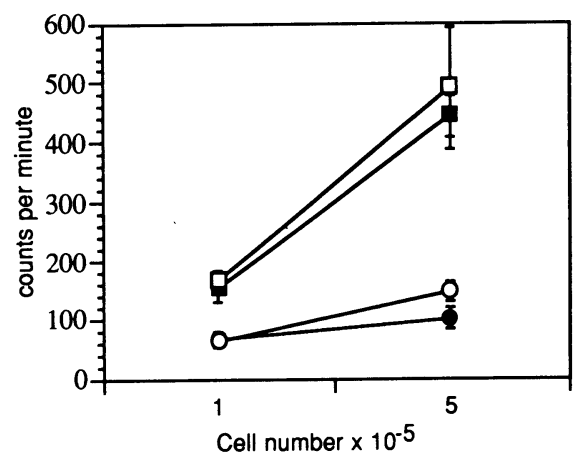

Figure 1. Ex vivo proliferation of lymphocytes from JHM virusinfected rats (6 dpi). Different amounts of lymphocytes from cervical lymph nodes and the central nervous system were assayed for proliferation by ${ }^{3} \mathrm{H}$-thymidine incorporation (lymph node cells $\square, \square$ and CNS-isolated lymphocytes $0, \bullet$ from LEW and BN rats respectively).

brain ${ }^{13}$. Consequently, we analyzed lymphocytes derived from CLNs of JHM infected BN and LEW rats. Figure 1 shows that these cells indeed do respond to the intracerebral virus infection by proliferation in a dose dependent manner when assayed ex vivo. After restimulation of CLN-lymphocytes with Con A the incorporation of ${ }^{3} \mathrm{H}$-thymidine was about 100-fold higher compared to lymphocytes from the brain (Fig. 2, right panel). Virusspecificity of this response was demonstrated by the fact that stimulation of CLN-cells by JHM-specific antigens resulted in a considerable increase of ${ }^{3} \mathrm{H}$-thymidine incorporation compared to the unrelated control antigen like KLH (Fig. 3, right panel). 


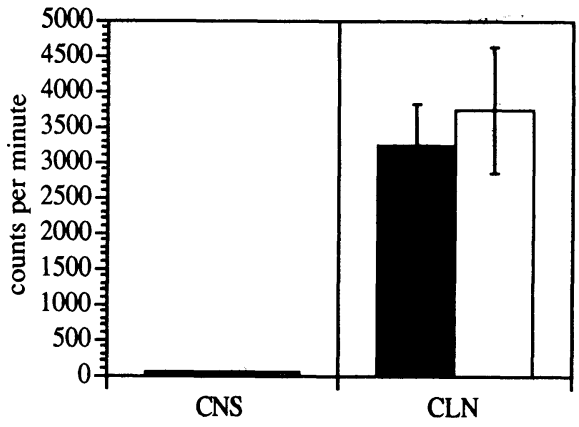

Figure 2. Mitogen-induced proliferation of lymphocytes from JHM virus-infected rats (6 dpi). Lymphocytes from the central nervous system (CNS) and cervical lymph nodes (CLN) of LEW $(\square)$ and BN (ם) rats were stimulated with concanavalin A. Proliferation was monitored by ${ }^{3} \mathrm{H}$-thymidine incorporation.

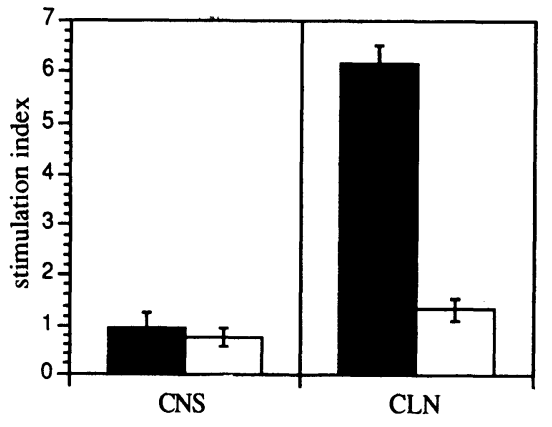

Figure 3. Antigen-induced proliferation of lymphocytes from JHM virus infected rats (6 dpi). Lymphocytes from the central nervous system (CNS) and cervical lymph nodes (CLN) of LEW $(\square)$ and $\mathrm{BN}(\square)$ rats were stimulated with control antigen (KLH = keyhole limpet hemocyanine) and JHM virus. Proliferation was monitored by ${ }^{3} \mathrm{H}$-thymidine incorporation. Virus-specific proliferation is expressed as stimulation index (counts per minute in virus-stimulated cells/counts per minute in KLH-stimulated cells) 
Interestingly, the antigen specific response in $\mathrm{BN}$ rats is much higher compared to LEW rats, especially early past infection. This result fits well to the finding that in BN rats virus-specific killing activity of brain-infiltrating $\mathrm{CD} 8^{+}$cells can be detected earlier compared to LEW rats ${ }^{4}$ as well as to previous reports by our laboratory that a virus-specific antibody response is recruited more rapid in BN rats 2,5 .

In summary, these data implicate that the subclinical course of the infection in BN rats might be explicable by a more rapid, vigorous and specific peripheral immune system response to the intracerebral JHM virus infection compared to LEW rats. The delay of the virus-specific response in LEW rats allows viral spread throughout the CNS resulting in a strong inflammatory $\mathrm{T}$-lymphocyte response. The accompanying paper in this volume ${ }^{14}$ shows that $\mathrm{CD}^{+}{ }^{+}$cells in the absence of $\mathrm{CD} 8^{+}$and $\mathrm{B}$-cells can significantly contribute to the severity of the clinical symptomatology in these rats. This opens up the prospect that CD4+ T-cells, which are essential regulators of T- and B-cell differentiation in secondary lymphoid organs, may fulfill other effector functions after they have passed the blood brain barrier. Currently we examine the expression of interleukins in CNS-derived leukocytes by reverse transcriptase polymerase chain reaction (RT-PCR) to shed more light on the yet unknown effector mechanisms of $\mathrm{CD}^{+}{ }^{+}$cells at the site of JHM virus infection.

\section{ACKNOWLEDGEMENTS}

This study was supported by the Bundesministerium für Forschung und Technologie of the Fed. Rep. of Germany (Grant no.: KI 01 8839-2).

We gratefully thank M. Zips and U. Sauer for expert technical assistance.

\section{REFERENCES}

1 R. Watanabe, H. Wege, \& V. ter Meulen, Lab. Invest. 57:375 (1987)

2 R. Dörries, R. Watanabe, H.Wege, \& V. ter Meulen, J. Neuroimmunol. 12:131 (1986)

3 R. Dörries, S. Schwender, H. Imrich, \& H. Harms, Immunology 12:539 (1991)

4 A. Hein, H. Imrich , S. Schwender,\& R. Dörries, same Volume

5 S. Schwender, H. Imrich,\& R. Dörries, Immunology 12:533 (1991)

6 H. Wege, A. Müller, \& V. ter Meulen, Lab. Invest. 41:217 (1978)

7 R.P. Arthur \& D. Mason, J. Exp. Med. 168:774 (1986)

8 F. Powrie \& D. Mason, J. Exp. Med. 169: 653 (1989)

9 J.D. Sedgwick \& R. Dörries, Seminars in THE NEUROSCIENCES 3: 93 (1991)

10 J.D. Sedgwick, S. Schwender, H. Imrich, R. Dörries, G.W. Butcher, \& V. ter Meulen, Proc. Natl. Acad. 88: 7438 (1991)

11 K. Ohmori, Y. Hong, M. Fujiwara, Y. Matsumoto, Lab. Invest. 66:54 (1992)

12 D. Nelson,. Strickland, \& P.G. Holt, Immunology 69: 476 (1991)

13 C. Harling-Berg, P.M. Knopf, J. Merriam, \& H.F. Cserr, J. Neuroimmunol. 25:185 (1989)

14 S. Schwender, A. Hein, H. Imrich, \& R. Dörries, same Volume 